\title{
General Convexity of Multidimensional Functions and Related Hermite- Hadamard Type Integral Inequalities
}

\author{
Çok Boyutlu Fonksiyonların Genelleştirilmiş Konveksliği ve İlgili Hermite-Hadamard Tipi \\ Integral Eşitsizlikleri
}

\author{
Fatma Buğlem YALÇIN*1,a , Nurgül OKUR ${ }^{2, b}$ \\ ${ }^{I}$ Department of Mathematics, Faculty of Science and Arts, Ordu University, Ordu, Turkey \\ ${ }^{2}$ Department of Statistics, Faculty of Science and Arts, Giresun University, Giresun, Turkey
}

\author{
• Geliş tarihi / Received: 14.10.2019 •Düzeltilerek geliş tarihi / Received in revised form: 30.11 .2019 • Kabul tarihi / Accepted: 06.12 .2019
}

\begin{abstract}
The basic goal is to investigate general convexity of multidimensional functions and derive several important inequalities associated with it's in this paper. For this reason, multidimensional general convex functions were firstly defined. Afterwards, some properties of these functions were mentioned. Accordingly, the relation of multidimensional general convex functions with other convex functions was established. Additionally, a generalization of HermiteHadamard type integral inequality was showed for two-dimensional general convex functions. Finally, HermiteHadamard type integral inequality for multidimensional general convex functions was verified and an explanatory example for this inequality was given in this study.
\end{abstract}

Keywords: Generalized Convexity, Hermite-Hadamard Inequality, Multidimensional General Convex Functions, nCoordinates

$\ddot{O} z$

Bu makalede temel amaç, çok boyutlu fonksiyonların genelleştirilmiş konveksliğini incelemek ve onunla ilgili bazı önemli eşitsizlikler elde etmektir. Bu nedenle ilk olarak çok boyutlu genelleştirilmiş konveks fonksiyonlar tanımlanmıştır. Devamında bu fonksiyonların bazı özelliklerinden bahsedilmiştir. Buna bağlı olarak, çok boyutlu genelleştirilmiş konveks fonksiyonlar ile diğer konveks fonksiyonların ilişsisi kurulmuştur. Ek olarak, iki boyutlu genelleş̧irilmiş konveks fonksiyonlar için Hermite-Hadamard tipli integral eşitsiziliği genelleştirilmişstir. Son olarak bu çalışmada, çok boyutlu genelleştirilmiş konveks için Hermite-Hadamard tipli integral eşitsizliği elde edilmiş ve bu eşitsizliği açıklayıcı bir örnek verilmişstir.

Anahtar Kelimeler: Genelleştirilmiş Konvekslik, Hermite-Hadamard Eşitsizliği, Çok Boyutlu Genelleştirilmiş Konveks Fonksiyonlar, n-Boyutlu Koordinatlar

\footnotetext{
*a Fatma Buğlem YALÇIN; yalcinfatmabuglem@ gmail.com, Tel: (0545) 60176 14, orcid.org/0000-0003-4276-1820

${ }^{\mathrm{b}}$ orcid.org/0000-0002-2544-7752
} 


\section{Introduction}

In the literature, Hermite-Hadamard type integral inequality for convex functions is known as the following inequality (Hadamard, 1893):

$$
f\left(\frac{t+s}{2}\right) \leq \frac{1}{s-t} \int_{t}^{s} f(x) d x \leq \frac{f(t)+f(s)}{2}
$$

In this context, many researchers studied on Hermite-Hadamard type integral inequality for $\varphi$ - convex functions (general convex functions) in literature (Martinez-Legaz and Singer, 1998; Syau and Lee, 2005; Dragomir, 2015; Shaikh et al., 2018; etc.).

For example, E-convexity is defined such that a function $f: \mathbb{R}^{n} \rightarrow \mathbb{R}$ is said to be E-convex on a set $M \subset \mathbb{R}^{n}$ iff there is a map $E: \mathbb{R}^{n} \rightarrow \mathbb{R}^{n}$ such that $M$ is an E-convex set and

$$
f(\lambda E(\alpha)+(1-\lambda) E(\beta)) \leq \lambda f(E(\alpha))+(1-\lambda) f(E(\beta))
$$

for each $\alpha, \beta \in M$ and $\lambda \in[0,1]$ (Youness, 1999). Youness's definition is introduced such that a function $f:[t, s] \subset \mathbb{R} \rightarrow \mathbb{R}$ is called general convex functions on the real number interval $[t, s]$, if the following inequality holds

$$
f(\lambda \varphi(\alpha)+(1-\lambda) \varphi(\beta)) \leq \lambda f(\varphi(\alpha))+(1-\lambda) f(\varphi(\beta))
$$

for all $\alpha, \beta \in[t, s], \lambda \in[0,1]$ and $\varphi:[t, s] \rightarrow[t, s], \varphi(t)<\varphi(s)$ is a function (Sarikaya et al., 2015). Moreover, Hermite-Hadamard type integral inequality for general convex functions is proved as follows (Cristescu, 2004):

$$
f\left(\frac{\varphi(t)+\varphi(s)}{2}\right) \leq \frac{1}{\varphi(t)-\varphi(s)} \int_{\varphi(t)}^{\varphi(s)} f(\alpha) d \alpha \leq \frac{f(\varphi(t))+f(\varphi(s))}{2}
$$

Also, the following general convex functions on the coordinates are showed (Set et al., 2014):

Let $\Delta:=\left[\vartheta_{1}, \omega_{1}\right] \times\left[\vartheta_{2}, \omega_{2}\right] \subseteq[0, \infty)^{2} ; \vartheta_{1}<\omega_{1}, \vartheta_{2}<\omega_{2} ; \varphi_{i}:\left[\vartheta_{i}, \omega_{i}\right] \rightarrow\left[\vartheta_{i}, \omega_{i}\right], i=1,2$ be a continuous function. A function $f: \Delta \rightarrow \mathbb{R}$ is called general convex functions on $\Delta$, if the following inequality holds

$$
\begin{gathered}
f\left(\lambda \varphi_{1}\left(\alpha_{1}\right)+(1-\lambda) \varphi_{1}\left(\alpha_{2}\right), \lambda \varphi_{2}\left(\beta_{1}\right)+(1-\lambda) \varphi_{2}\left(\beta_{2}\right)\right) \\
\quad \leq \lambda f\left(\varphi_{1}\left(\alpha_{1}\right), \varphi_{2}\left(\beta_{1}\right)\right)+(1-\lambda) f\left(\varphi_{1}\left(\alpha_{2}\right), \varphi_{2}\left(\beta_{2}\right)\right)
\end{gathered}
$$

for all $\left(\alpha_{1}, \beta_{1}\right),\left(\alpha_{2}, \beta_{2}\right) \in \Delta$ and $\lambda \in[0,1]$. If the above inequality is reversed then $f$ is said to be $\varphi$-concave on $\Delta$.

Other definition of general convex functions is defined as follows (Set et al., 2014):

A function $f: \Delta \rightarrow \mathbb{R}$ is called coordinated $\varphi$-convex on $\Delta$, if the following partial mappings $f_{\varphi_{2}}:\left[\vartheta_{1}, \omega_{1}\right] \rightarrow$ $\mathbb{R}, f_{\varphi_{2}}(\vartheta):=f\left(\vartheta, \varphi_{2}\right)$ and $f_{\varphi_{1}}:\left[\vartheta_{2}, \omega_{2}\right] \rightarrow \mathbb{R}, f_{\varphi_{1}}(\omega):=f\left(\varphi_{1}, \omega\right)$ are defined $\varphi$-convex for all $\varphi_{1} \in$ $\left[\vartheta_{1}, \omega_{1}\right]$ and $\varphi_{2} \in\left[\vartheta_{2}, \omega_{2}\right]$.Then 


$$
\begin{gathered}
\quad \frac{f\left(\frac{\varphi_{1}\left(\vartheta_{1}\right)+\varphi_{1}\left(\omega_{1}\right)}{2}, \frac{\varphi_{2}\left(\vartheta_{2}\right)+\varphi_{2}\left(\omega_{2}\right)}{2}\right)}{\varphi_{1}\left(\omega_{1}\right)} \int_{\left.\varphi_{1}\left(\omega_{1}\right)-\varphi_{1}\left(\vartheta_{1}\right)\right)}^{\int_{\varphi_{1}\left(\vartheta_{1)}\right.}} f\left(\alpha, \frac{\varphi_{2}\left(\vartheta_{2}\right)+\varphi_{2}\left(\vartheta_{2}\right)}{2}\right) d \alpha \\
+\frac{1}{2\left(\varphi_{2}\left(\omega_{2}\right)-\varphi_{2}\left(\vartheta_{2}\right)\right)} \int_{\varphi_{2}\left(\vartheta_{2}\right)}^{\left.\varphi_{2}\right)} f\left(\frac{\varphi_{1}\left(\vartheta_{1}\right)+\varphi_{1}\left(\omega_{1}\right)}{2}, \beta\right) d \beta \\
\leq \frac{1}{\left(\varphi_{1}\left(\omega_{1}\right)-\varphi_{1}\left(\vartheta_{1}\right)\right)\left(\varphi_{2}\left(\omega_{2}\right)-\varphi_{2}\left(\vartheta_{2}\right)\right)} \int_{\varphi\left(\vartheta_{1}\left(\vartheta_{1}\right)\right.}^{\varphi_{2}\left(\omega_{2}\right)} \int_{\varphi_{2}\left(\vartheta_{2}\right)}^{\varphi_{2}} f(\alpha, \beta) d \alpha d \beta \\
\leq \frac{1}{4\left(\varphi_{1}\left(\omega_{1}\right)-\varphi_{1}\left(\vartheta_{1}\right)\right)} \int_{\varphi\left(\vartheta_{1}\right)}^{\omega_{\varphi\left(\omega_{2}\right)}}\left[f\left(\alpha, \varphi_{2}\left(\vartheta_{2}\right)\right)+f\left(\alpha, \varphi_{2}\left(\omega_{2}\right)\right)\right] d \alpha \\
+\frac{1}{4\left(\varphi_{2}\left(\omega_{2}\right)-\varphi_{2}\left(\vartheta_{2}\right)\right)} \int_{\varphi\left(\omega_{1}\right)}\left[f\left(\varphi_{1}\left(\vartheta_{1}\right), \beta\right)+f\left(\varphi_{1}\left(\omega_{1}\right), \beta\right)\right] d \beta \\
\leq \frac{1}{4}\left[f\left(\varphi_{1}\left(\vartheta_{1}\right), \varphi_{2}\left(\vartheta_{2}\right)\right)+f\left(\varphi_{1}\left(\vartheta_{1}\right), \varphi_{2}\left(\omega_{2}\right)\right)\right. \\
\left.+f\left(\varphi_{1}\left(\omega_{1}\right), \varphi_{2}\left(\vartheta_{2}\right)\right)+f\left(\varphi_{1}\left(\omega_{1}\right), \varphi_{2}\left(\omega_{2}\right)\right)\right]
\end{gathered}
$$

Recently, some generalized inequalities about two-dimensional general convex functions are verified (Yalçın, 2019). There are many studies on generalization of convexity of functions. Hadamard's inequality for convex functions on the coordinates in a rectangle from the plane is obtained (Dragomir, 2001). Hadamard-type inequalities for $h$-convex functions on the coordinates are derived (Latif and Alomari, 2009). Hadamard-type inequalities for $\mathrm{p}$-convex stochastic processes are obtained (Okur et al., 2019). Moreover, Hermite-Hadamard type integral inequality for two-dimensional operator Harmonically convex functions is extended (Okur and Yalçın, 2019).

There are also many studies on multidimensional convex functions in literature. Hermite-Hadamard type integral inequality for multidimensional convex functions is investigated (De la $\mathrm{Cal}$ and Carcamo, 2006). Hermite-Hadamard type integral inequality for $s$ - multidimensional convex functions is studied (Elahi et al., 2015). HermiteHadamard type integral inequality for Harmonically multidimensional convex functions is verified (Viloria and Cortez, 2018). Nowadays, multidimensional general convexity for stochastic processes is defined and Hermite-Hadamard type integral inequality associated with its is obtained (Okur, 2019).

In the light of such information, we investigated multidimensional general convexity for functions in this study.

\section{Results and Discussion}

In this section, we identified multidimensional general convex functions and proved Hermite-Hadamard type integral inequality for these functions. Let $\varphi_{i}:\left[\vartheta_{i}, \omega_{i}\right] \rightarrow\left[\vartheta_{i}, \omega_{i}\right]$ be a continuous increasing function and for $i=1,2, \ldots, n, n \geq 2$

$$
\begin{gathered}
\kappa^{n}:=\prod_{i=1}^{n}\left[\vartheta_{i}, \omega_{i}\right] \subseteq[0, \infty)^{n} ; \\
\phi_{i}^{+}:=\varphi_{i}\left(\vartheta_{i}\right)+\varphi_{i}\left(\omega_{i}\right) ; \phi_{i}^{-}:=\varphi_{i}\left(\omega_{i}\right)-\varphi_{i}\left(\vartheta_{i}\right) \text { such that } \varphi_{i}\left(\omega_{i}\right)<\varphi_{i}\left(\vartheta_{i}\right) ; \\
\boldsymbol{\varphi}(\boldsymbol{\alpha}):=\left(\bigwedge_{k=1}^{n} \varphi_{k}\left(\alpha_{k}\right)\right) \equiv\left(\varphi_{1}\left(\alpha_{1}\right), \ldots, \varphi_{n}\left(\alpha_{n}\right)\right) \equiv \varphi\left(\alpha_{1}, \ldots, \alpha_{n}\right) \in \kappa^{n} .
\end{gathered}
$$


Let us give definition of multidimensional general convex functions:

Definition 2.1. Suppose that the function $\varphi: \kappa^{n} \rightarrow \xi^{n}$ is a continuous increasing. Then $\psi: \kappa^{n} \rightarrow \mathbb{R}$ is called multidimensional general convex functions if

$$
\psi(\theta \boldsymbol{\varphi}(\boldsymbol{\alpha})+(1-\theta) \boldsymbol{\varphi}(\boldsymbol{\beta})) \leq \theta \psi(\boldsymbol{\varphi}(\boldsymbol{\alpha}))+(1-\theta) \psi(\boldsymbol{\varphi}(\boldsymbol{\beta})),
$$

for all $\theta \in[0,1]$.

By Definition 2.1, we have

(i) general convex functions for $n=1$,

(ii) two-dimensional general convex functions for $n=2$,

(iii) multidimensional convex functions if $\varphi$ is an identity function,

(iv) convex functions if $\varphi$ is an identity function and for $n=1$.

Definition 2.2. $\psi: \xi^{n} \rightarrow \mathbb{R}$ is called multidimensional general convex functions on $\xi^{n}$ if the following partial functions $\psi_{\varphi_{i}\left(\alpha_{i}\right)}^{i}:\left[\vartheta_{i}, \omega_{i}\right] \rightarrow \mathbb{R}$ are general convex on $\left[\vartheta_{i}, \omega_{i}\right]$

$$
\psi_{\varphi_{i}\left(\alpha_{i}\right)}^{i}(\alpha):=\psi\left(\bigwedge_{k=1}^{i-1} \varphi_{k}\left(\alpha_{k}\right), \alpha, \Lambda_{k=i+1}^{n} \varphi_{k}\left(\alpha_{k}\right)\right)
$$

for all $\psi_{\varphi_{i}\left(\alpha_{i}\right)}^{i} \in\left[\vartheta_{i}, \omega_{i}\right], i=1,2, \ldots, n, n \geq 2$

Lemma 2.1. Every general convex functions $\psi: \kappa^{n} \rightarrow \mathbb{R}$ is general convex on $n$-coordinates, not the other way round.

Proof. Let $\psi: \kappa^{n} \rightarrow \mathbb{R}$ be a multidimensional general convex functions. Using the definition of $\psi_{\varphi_{n}\left(\alpha_{n}\right)}^{i}$, we get

$$
\begin{gathered}
\psi_{\varphi_{n}\left(\alpha_{n}\right)}^{i}(\theta \varphi(\alpha)+(1-\theta) \varphi(\beta)) \\
=\psi\left(\Lambda_{k=1}^{i-1} \varphi_{k}\left(\alpha_{k}\right), \theta \varphi(\alpha)+(1-\theta) \varphi(\beta), \Lambda_{k=i+1}^{n} \varphi_{k}\left(\alpha_{k}\right)\right) \\
\leq \theta \psi\left(\bigwedge_{k=1}^{i-1} \varphi_{k}\left(\alpha_{k}\right), \varphi(\alpha), \wedge_{k=i+1}^{n} \varphi_{k}\left(\alpha_{k}\right)\right) \\
+(1-\theta) \psi\left(\Lambda_{k=1}^{i-1} \varphi_{k}\left(\alpha_{k}\right), \varphi(\beta), \wedge_{k=i+1}^{n} \varphi_{k}\left(\alpha_{k}\right)\right) \\
=\theta \psi_{\varphi_{n}\left(\alpha_{n}\right)}^{i}(\varphi(\alpha))+(1-\theta) \psi_{\varphi_{n}\left(\alpha_{n}\right)}^{i}(\varphi(\beta)) .
\end{gathered}
$$

But then, suppose that $\psi:[0,1]^{n} \rightarrow \mathbb{R}$;

$$
\psi(\boldsymbol{\varphi}(\boldsymbol{\alpha})):=\varphi_{1}\left(\alpha_{1}\right) \varphi_{2}\left(\alpha_{2}\right) \ldots \varphi_{n}\left(\alpha_{n}\right)
$$

This function is clearly a multidimensional general convex functions. But for $\boldsymbol{\varphi}(\boldsymbol{\alpha})=(1,1, \ldots, 0), \boldsymbol{\varphi}(\boldsymbol{\beta})=$ $(0,1, \ldots, 1) \in[0,1]^{n}$, we have

$$
\psi(\theta \boldsymbol{\varphi}(\boldsymbol{\alpha})+(1-\theta) \boldsymbol{\varphi}(\boldsymbol{\beta}))=\psi(\theta, 1,1, \ldots,(1-\theta))=\theta(1-\theta)
$$




$$
\theta \psi(\boldsymbol{\varphi}(\boldsymbol{\alpha}))+(1-\theta) \psi(\boldsymbol{\varphi}(\boldsymbol{\beta}))=\theta .0+(1-\theta) .0=0 .
$$

This gives $\psi(\theta \boldsymbol{\varphi}(\boldsymbol{\alpha})+(1-\theta) \boldsymbol{\varphi}(\boldsymbol{\beta}))>\theta \psi(\boldsymbol{\varphi}(\boldsymbol{\alpha}))+(1-\theta) \psi(\boldsymbol{\varphi}(\boldsymbol{\beta}))$ for all $\theta \in[0,1]$, namely, $\psi$ is not general convex on $[0,1]^{n}$.

Remark 2.1. If $\psi: \xi^{n} \rightarrow \mathbb{R}$ is multidimensional general convex functions, then $\psi_{\varphi_{n}\left(\alpha_{n}\right)}^{i}:\left[\vartheta_{i}, \omega_{i}\right] \rightarrow \mathbb{R}$ is general convex functions such that

$$
\begin{gathered}
\psi_{\varphi_{n}\left(\alpha_{n}\right)}^{i}\left(\frac{\phi_{i}^{+}}{2}\right) \leq \frac{1}{\phi_{i}^{-}} \int_{\varphi_{i}\left(\vartheta_{i}\right)}^{\varphi_{i}\left(\omega_{i}\right)} \psi_{\varphi_{n}\left(\alpha_{n}\right)}^{i}\left(\alpha_{i}\right) d \alpha_{i} \\
\leq \frac{\psi_{\varphi_{n}\left(\alpha_{n}\right)}^{i}\left(\varphi_{i}\left(\vartheta_{i}\right)\right)+\psi_{\varphi_{n}\left(\alpha_{n}\right)}^{i}\left(\varphi_{i}\left(\omega_{i}\right)\right)}{2} .
\end{gathered}
$$

Theorem 2.1. Let $\psi: \xi^{n} \rightarrow \mathbb{R}$ be a multidimensional general convex functions. Then

$$
\begin{gathered}
\sum_{i=1}^{n} \frac{1}{\phi_{i}^{-}} \int_{\varphi_{i}\left(\vartheta_{i}\right)}^{\varphi_{i}\left(\omega_{i}\right)}\left(\psi_{\varphi_{n}\left(\vartheta_{n}\right)}^{i}\left(\alpha_{i}\right)+\psi_{\varphi_{n}\left(\omega_{n}\right)}^{i}\left(\alpha_{i}\right)\right) d \alpha_{i} \\
\leq \frac{n}{2}[\psi(\boldsymbol{\varphi}(\boldsymbol{\vartheta}))+\psi(\boldsymbol{\varphi}(\boldsymbol{\omega}))]+\frac{1}{2} \sum_{i=1}^{n}\left[\psi_{\varphi_{n}\left(\vartheta_{n}\right)}^{i}\left(\varphi_{i}\left(\vartheta_{i}\right)\right)+\psi_{\varphi_{n}\left(\omega_{n}\right)}^{i}\left(\varphi_{i}\left(\omega_{i}\right)\right)\right] .
\end{gathered}
$$

Proof. Using the left hand of (1) by $\psi_{\varphi_{n}\left(\vartheta_{n}\right)}^{i}\left(\varphi_{i}\left(\vartheta_{i}\right)\right)=\psi(\boldsymbol{\varphi}(\boldsymbol{\vartheta}))$ and $\psi_{\varphi_{n}\left(\omega_{n}\right)}^{i}\left(\varphi_{i}\left(\omega_{i}\right)\right)=\psi(\boldsymbol{\varphi}(\boldsymbol{\omega}))$ for each $i=1, \ldots, n$, then

$$
\begin{gathered}
\frac{1}{\phi_{i}^{-}} \int_{\varphi_{i}\left(\vartheta_{i}\right)}^{\varphi_{i}\left(\omega_{i}\right)} \psi_{\varphi_{n}\left(\vartheta_{n}\right)}^{i}\left(\alpha_{i}\right) d \alpha_{i} \\
\leq \frac{\psi_{\varphi_{n}\left(\vartheta_{n}\right)}^{i}\left(\varphi_{i}\left(\vartheta_{i}\right)\right)+\psi_{\varphi_{n}\left(\vartheta_{n}\right)}^{i}\left(\varphi_{i}\left(\omega_{i}\right)\right)}{2} \leq \frac{\psi(\boldsymbol{\varphi}(\boldsymbol{\vartheta}))+\psi_{\varphi_{n}\left(\vartheta_{n}\right)}^{i}\left(\varphi_{i}\left(\omega_{i}\right)\right)}{2} \\
\leq \frac{\frac{1}{\phi_{i}^{-}} \int_{\varphi_{i}\left(\vartheta_{i}\right)}^{\varphi_{i}\left(\omega_{i}\right)} \psi_{\varphi_{n}\left(\omega_{n}\right)}^{i}\left(\alpha_{i}\right) d \alpha_{i}}{2} \leq \frac{\psi_{\varphi_{n}\left(\omega_{n}\right)}^{i}\left(\varphi_{i}\left(\vartheta_{i}\right)\right)+\psi_{\varphi_{n}\left(\omega_{n}\right)}^{i}\left(\varphi_{i}\left(\omega_{i}\right)\right)}{2}\left(\varphi_{i}\left(\vartheta_{i}\right)\right)+\psi(\boldsymbol{\varphi}(\boldsymbol{\omega})) \\
2
\end{gathered}
$$

Aggregating of the above inequalities by integrating on $\left[\varphi_{i}\left(\vartheta_{i}\right), \varphi_{i}\left(\omega_{i}\right)\right]$

$$
\begin{gathered}
\frac{1}{\phi_{i}^{-}} \int_{\varphi_{i}\left(\vartheta_{i}\right)}^{\varphi_{i}\left(\omega_{i}\right)}\left[\psi_{\varphi_{n}\left(\vartheta_{n}\right)}^{i}\left(\alpha_{i}\right)+\psi_{\varphi_{n}\left(\omega_{n}\right)}^{i}\left(\alpha_{i}\right)\right] d \alpha_{i} \\
\leq \frac{\psi(\boldsymbol{\varphi}(\boldsymbol{\vartheta}))+\psi_{\varphi_{n}\left(\vartheta_{n}\right)}^{i}\left(\varphi_{i}\left(\omega_{i}\right)\right)+\psi(\boldsymbol{\varphi}(\boldsymbol{\omega}))+\psi_{\varphi_{n}\left(\omega_{n}\right)}^{i}\left(\varphi_{i}\left(\vartheta_{i}\right)\right)}{2} .
\end{gathered}
$$

Taking summation from 1 to $n$, this completes the proof.

Theorem 2.2. Let $\psi: \xi^{n} \rightarrow \mathbb{R}$ be a multidimensional general convex functions. Then

$$
\sum_{i=1}^{n-1} \psi\left(\Lambda_{k=1}^{i-1} \varphi_{k}\left(\alpha_{k}\right), \frac{\phi_{i}^{+}}{2}, \frac{\phi_{i+1}^{+}}{2}, \Lambda_{k=i+2}^{n} \varphi_{k}\left(\alpha_{k}\right),\right)
$$




$$
\begin{gathered}
\leq \sum_{i=1}^{n-1} \frac{1}{\phi_{i}^{-}} \int_{\varphi_{i}\left(\vartheta_{i}\right)}^{\varphi_{i}\left(\omega_{i}\right)} \psi_{\varphi_{i+1}\left(\alpha_{n}\right)}^{i+1}\left(\frac{\phi_{i+1}^{+}}{2}\right) d \alpha_{i} \\
\leq \sum_{i=1}^{n-1} \frac{1}{\phi_{i}^{-} \phi_{i+1}^{-}} \int_{\varphi_{i}\left(\vartheta_{i}\right)}^{\varphi_{i}\left(\omega_{i}\right)} \int_{\varphi_{i+1}\left(\vartheta_{i+1}\right)}^{\varphi_{i+1}\left(\omega_{i+1}\right)} \psi_{\varphi_{i+1}\left(\alpha_{n}\right)}^{i+1}\left(\alpha_{i+1}\right) d \alpha_{i+1} d \alpha_{i} \\
\leq \sum_{i=1}^{n-1} \frac{1}{2 \phi_{i}^{-}} \int_{\varphi_{i}\left(\vartheta_{i}\right)}^{\varphi_{i}\left(\omega_{i}\right)}\left[\psi_{\varphi_{i+1}\left(\alpha_{n}\right)}^{i+1}\left(\varphi\left(\vartheta_{i+1}\right)\right)+\psi_{\varphi_{i+1}\left(\alpha_{n}\right)}^{i+1}\left(\varphi\left(\omega_{i+1}\right)\right)\right] d \alpha_{i} \\
\leq \frac{1}{4} \sum_{i=1}^{n-1}\left[\begin{array}{c}
\psi\left(\bigwedge_{k=1}^{i-1} \varphi_{k}\left(\alpha_{k}\right), \varphi_{i}\left(\vartheta_{i}\right), \varphi_{i+1}\left(\vartheta_{i+1}\right), \bigwedge_{k=i+2}^{n} \varphi_{k}\left(\alpha_{k}\right)\right) \\
+\psi\left(\bigwedge_{k=1}^{i-1} \varphi_{k}\left(\alpha_{k}\right), \varphi_{i}\left(\omega_{i}\right), \varphi_{i+1}\left(\vartheta_{i+1}\right), \bigwedge_{k=i+2}^{n} \varphi_{k}\left(\alpha_{k}\right)\right) \\
+\psi\left(\bigwedge_{k=1}^{i-1} \varphi_{k}\left(\alpha_{k}\right), \varphi_{i}\left(\vartheta_{i}\right), \varphi_{i+1}\left(\omega_{i+1}\right), \bigwedge_{k=i+2}^{n} \varphi_{k}\left(\alpha_{k}\right)\right) \\
+\psi\left(\bigwedge_{k=1}^{i-1} \varphi_{k}\left(\alpha_{k}\right), \varphi_{i}\left(\omega_{i}\right), \varphi_{i+1}\left(\omega_{i+1}\right), \bigwedge_{k=i+2}^{n} \varphi_{k}\left(\alpha_{k}\right)\right)
\end{array}\right]
\end{gathered}
$$

Proof. Using (1) by $\psi_{\varphi_{i+1}\left(\alpha_{n}\right)}^{i+1}$, then

$$
\begin{gathered}
\psi_{\varphi_{i+1}\left(\alpha_{n}\right)}^{i+1}\left(\frac{\phi_{i+1}^{+}}{2}\right) \leq \frac{1}{\phi_{i+1}^{-}} \int_{\varphi_{i+1}\left(\vartheta_{i+1}\right)}^{\varphi_{i+1}\left(\omega_{i+1}\right)} \psi_{\varphi_{i+1}\left(\alpha_{n}\right)}^{i+1}\left(\alpha_{i+1}\right) d \alpha_{i+1} \\
\leq \frac{\psi_{\varphi_{i+1}\left(\alpha_{n}\right)}^{i+1}\left(\varphi_{i+1}\left(\vartheta_{i+1}\right)\right)+\psi_{\varphi_{i+1}\left(\alpha_{n}\right)}^{i+1}\left(\varphi_{i+1}\left(\omega_{i+1}\right)\right)}{2} .
\end{gathered}
$$

Integrating all of sides of the above inequalities on $\left[\varphi_{i}\left(\vartheta_{i}\right), \varphi_{i}\left(\omega_{i}\right)\right]$

$$
\begin{gathered}
\frac{1}{\phi_{i}^{-}} \int_{\varphi_{i}\left(\vartheta_{i}\right)}^{\varphi_{i}\left(\omega_{i}\right)} \psi_{\varphi_{i+1}\left(\alpha_{n}\right)}^{i+1}\left(\frac{\phi_{i+1}^{+}}{2}\right) d \alpha_{i} \\
\leq \frac{1}{\phi_{i}^{-} \phi_{i+1}^{-}} \int_{\varphi_{i}\left(\vartheta_{i}\right)}^{\varphi_{i}\left(\omega_{i}\right)} \int_{\varphi_{i+1}\left(\vartheta_{i+1}\right)}^{\varphi_{i+1}\left(\omega_{i+1}\right)} \psi_{\varphi_{i+1}\left(\alpha_{n}\right)}^{i+1}\left(\alpha_{i+1}\right) d \alpha_{i+1} d \alpha_{i} \\
\leq \frac{1}{2 \phi_{i}^{-}} \int_{\varphi_{i}\left(\vartheta_{i}\right)}^{\varphi_{i}\left(\omega_{i}\right)}\left(\psi_{\varphi_{i+1}\left(\alpha_{n}\right)}^{i+1}\left(\varphi_{i+1}\left(\vartheta_{i+1}\right)\right)+\psi_{\varphi_{i+1}\left(\alpha_{n}\right)}^{i+1}\left(\varphi_{i+1}\left(\omega_{i+1}\right)\right)\right) d \alpha_{i} .
\end{gathered}
$$

Applying Hermite-Hadamard type integral inequality to the left hand of (4) for each $i \in\{1, \ldots, n-1\}$

$$
\psi\left(\Lambda_{k=1}^{i-1} \varphi_{k}\left(\alpha_{k}\right), \frac{\phi_{i}^{+}}{2}, \frac{\phi_{i+1}^{+}}{2}, \Lambda_{k=i+2}^{n} \varphi_{k}\left(\alpha_{k}\right)\right) \leq \frac{1}{\phi_{i}^{-}} \int_{\varphi_{i}\left(\vartheta_{i}\right)}^{\varphi_{i}\left(\omega_{i}\right)} \psi_{\varphi_{i+1}\left(\alpha_{n}\right)}^{i+1}\left(\frac{\phi_{i+1}^{+}}{2}\right) d \alpha_{i}
$$

and also applying Hermite-Hadamard type integral inequality to the right hand of (4)

$$
\begin{gathered}
\frac{1}{2}\left[\frac{1}{\phi_{i}^{-}} \int_{\varphi_{i}\left(\vartheta_{i}\right)}^{\varphi_{i}\left(\omega_{i}\right)} \psi_{\varphi_{i+1}\left(\alpha_{n}\right)}^{i+1}\left(\varphi_{i+1}\left(\vartheta_{i+1}\right)\right) d \alpha_{i}+\frac{1}{\phi_{i}^{-}} \int_{\varphi_{i}\left(\vartheta_{i}\right)}^{\varphi_{i}\left(\omega_{i}\right)} \psi_{\varphi_{i+1}\left(\alpha_{n}\right)}^{i+1}\left(\varphi_{i+1}\left(\omega_{i+1}\right)\right) d \alpha_{i}\right] \\
\leq \frac{1}{4}\left[\begin{array}{c}
\psi\left(\bigwedge_{k=1}^{i-1} \varphi_{k}\left(\alpha_{k}\right), \varphi_{i}\left(\vartheta_{i}\right), \varphi_{i+1}\left(\vartheta_{i+1}\right), \bigwedge_{k=i+2}^{n} \varphi_{k}\left(\alpha_{k}\right)\right) \\
+\psi\left(\bigwedge_{k=1}^{i-1} \varphi_{k}\left(\alpha_{k}\right), \varphi_{i}\left(\omega_{i}\right), \varphi_{i+1}\left(\vartheta_{i+1}\right), \bigwedge_{k=i+2}^{n} \varphi_{k}\left(\alpha_{k}\right)\right) \\
+\psi\left(\bigwedge_{k=1}^{i-1} \varphi_{k}\left(\alpha_{k}\right), \varphi_{i}\left(\vartheta_{i}\right), \varphi_{i+1}\left(\omega_{i+1}\right), \bigwedge_{k=i+2}^{n} \varphi_{k}\left(\alpha_{k}\right)\right) \\
+\psi\left(\bigwedge_{k=1}^{i-1} \varphi_{k}\left(\alpha_{k}\right), \varphi_{i}\left(\omega_{i}\right), \varphi_{i+1}\left(\omega_{i+1}\right), \bigwedge_{k=i+2}^{n} \varphi_{k}\left(\alpha_{k}\right)\right)
\end{array}\right]
\end{gathered}
$$

for each $i \in\{1, \ldots, n-1\}$. After using the inequalities (5) and (6) in (4) and then taking summation from 1 to $n-1$, we have (3).

Remark 2.2. Using Theorem 2.2 for $n=1$, then the classical Hermite-Hadamard type integral inequality for two-dimensional general convex functions. 
Theorem 2.3. Let $\psi: \xi^{n} \rightarrow \mathbb{R}$ be a multidimensional general convex functions. Then

where

$$
\begin{gathered}
\psi\left(\frac{\phi_{1}^{+}}{2}, \ldots, \frac{\phi_{n-1}^{+}}{2}, \frac{\phi_{n}^{+}}{2}\right) \\
\leq \frac{1}{\prod_{i=1}^{n} \phi_{i}^{-}} \int_{\varphi_{1}\left(\vartheta_{1}\right)}^{\varphi_{1}\left(\omega_{1}\right)} \ldots \int_{\varphi_{n}\left(\vartheta_{n}\right)}^{\varphi_{n}\left(\omega_{n}\right)} \psi\left(\alpha_{1}, \ldots, \alpha_{n}\right) d \alpha_{n} \ldots d \alpha_{1} \\
\leq \frac{1}{2^{n}} \sum_{\xi \in \tau_{i}(n)} \psi(\xi \boldsymbol{\varphi}(\boldsymbol{\vartheta})+(\mathbf{1}-\xi) \boldsymbol{\varphi}(\boldsymbol{\omega}))
\end{gathered}
$$

$\tau_{i}(n):=\left\{\xi \in \mathbb{N}_{0}^{n}: \xi_{i} \leq 1,|\xi|=n+1-i, i=1, \ldots, n+1\right\} ;$

$|\xi|:=\xi_{1}+\cdots+\xi_{n} \in \mathbb{N} ; \xi \boldsymbol{\varphi}(\boldsymbol{\vartheta}):=\left(\xi_{1} \varphi_{1}\left(\vartheta_{1}\right), \ldots, \xi_{n} \varphi_{n}\left(\vartheta_{n}\right)\right) \in \mathbb{N}_{0}^{n}$.

Proof. Using (1), we get the following inequality for $\psi_{\varphi_{n}\left(\alpha_{n}\right)}^{n}$

$$
\begin{gathered}
\psi_{\varphi_{n}\left(\alpha_{n}\right)}^{n}\left(\frac{\phi_{n}^{+}}{2}\right) \leq \frac{1}{\phi_{n}^{-}} \int_{\varphi_{n}\left(\vartheta_{n}\right)}^{\varphi_{n}\left(\omega_{n}\right)} \psi_{\varphi_{n}\left(\alpha_{n}\right)}^{n}\left(\alpha_{n}\right) d \alpha_{n} \\
\leq \frac{\psi_{\varphi_{n}\left(\alpha_{n}\right)}^{n}\left(\varphi_{n}\left(\vartheta_{n}\right)\right)+\psi_{\varphi_{n}\left(\alpha_{n}\right)}^{n}\left(\varphi_{n}\left(\omega_{n}\right)\right)}{2} .
\end{gathered}
$$

Also, using the same method in the proof of Theorem 2.2 by the inequality (8), then

$$
\begin{array}{r}
\psi\left(\Lambda_{k=1}^{n-2} \varphi_{k}\left(\alpha_{k}\right), \frac{\phi_{n-1}^{+}}{2}, \frac{\phi_{n}^{+}}{2}\right) \leq \frac{1}{\phi_{n-1}^{-} \phi_{n}^{-}} \int_{\varphi_{n-1}\left(\vartheta_{n}\right)}^{\varphi_{n-1}\left(\omega_{n}\right)} \int_{\varphi_{n}\left(\vartheta_{n}\right)}^{\varphi_{n}\left(\omega_{n}\right)} \psi_{\varphi_{n}\left(\alpha_{n}\right)}^{n}\left(\alpha_{n}\right) d \alpha_{n} d \alpha_{n-1} \\
\leq \frac{1}{2^{2}}\left[\begin{array}{c}
\psi\left(\Lambda_{k=1}^{n-2} \varphi_{k}\left(\alpha_{k}\right), \varphi_{n-1}\left(\vartheta_{n-1}\right), \varphi_{n}\left(\vartheta_{n}\right)\right)+\psi\left(\Lambda_{k=1}^{n-2} \varphi_{k}\left(\alpha_{k}\right), \varphi_{n-1}\left(\omega_{n-1}\right), \varphi_{n}\left(\vartheta_{n}\right)\right) \\
+\psi\left(\Lambda_{k=1}^{n-2} \varphi_{k}\left(\alpha_{k}\right), \varphi_{n-1}\left(\vartheta_{n-1}\right), \varphi_{n}\left(\omega_{n}\right)\right)+\psi\left(\Lambda_{k=1}^{n-2} \varphi_{k}\left(\alpha_{k}\right), \varphi_{n-1}\left(\omega_{n-1}\right), \varphi_{n}\left(\omega_{n}\right)\right)
\end{array}\right] .
\end{array}
$$

Integrating (9) on $\left[\varphi_{n-2}\left(\vartheta_{n-2}\right), \varphi_{n-2}\left(\omega_{n-2}\right)\right]$

$$
\begin{gathered}
\frac{1}{\phi_{n-2}^{-}} \int_{\varphi_{n-2}\left(\vartheta_{n-2}\right)}^{\varphi_{n-2}\left(\omega_{n-2}\right)} \psi\left(\Lambda_{k=1}^{n-2} \varphi_{k}\left(\alpha_{k}\right), \frac{\phi_{n-1}^{+}}{2}, \frac{\phi_{n}^{+}}{2}\right) d \alpha_{n-2} \\
\leq \frac{1}{\prod_{i=n-2}^{n} \phi_{i}^{-}} \int_{\varphi_{n-2}\left(\vartheta_{n-2}\right)}^{\varphi_{n-2}\left(\omega_{n-2}\right)} \int_{\varphi_{n-1}\left(\vartheta_{n-1}\right)}^{\varphi_{n-1}\left(\omega_{n-1}\right)} \int_{\varphi_{n}\left(\vartheta_{n}\right)}^{\varphi_{n}\left(\omega_{n}\right)} \psi_{\varphi_{n}\left(\alpha_{n}\right)}^{n}\left(\alpha_{n}\right) d \alpha_{n} d \alpha_{n-1} d \alpha_{n-2} \\
\leq \frac{1}{\phi_{n-2}^{-}} \int_{\varphi_{n-2}\left(\vartheta_{n-2}\right)}^{\varphi_{n-2}\left(\omega_{n-2}\right)} \frac{1}{2^{2}}\left[\begin{array}{c}
\psi\left(\Lambda_{k=1}^{n-2} \varphi_{k}\left(\alpha_{k}\right), \varphi_{n-1}\left(\vartheta_{n-1}\right), \varphi_{n}\left(\vartheta_{n}\right)\right) \\
+\psi\left(\Lambda_{k=1}^{n-2} \varphi_{k}\left(\alpha_{k}\right), \varphi_{n-1}\left(\omega_{n-1}\right), \varphi_{n}\left(\vartheta_{n}\right)\right) \\
+\psi\left(\Lambda_{k=1}^{n-2} \varphi_{k}\left(\alpha_{k}\right), \varphi_{n-1}\left(\vartheta_{n-1}\right), \varphi_{n}\left(\omega_{n}\right)\right) \\
+\psi\left(\Lambda_{k=1}^{n-2} \varphi_{k}\left(\alpha_{k}\right), \varphi_{n-1}\left(\omega_{n-1}\right), \varphi_{n}\left(\omega_{n}\right)\right)
\end{array}\right] d \alpha_{n-2} .
\end{gathered}
$$

Again using the same method in the proof of Theorem 2.2 by the inequality (10), then

$$
\begin{gathered}
\psi\left(\Lambda_{k=1}^{n-3} \varphi_{k}\left(\alpha_{k}\right), \frac{\phi_{n-2}^{+}}{2}, \frac{\phi_{n-1}^{+}}{2}, \frac{\phi_{n}^{+}}{2}\right) \\
\leq \frac{1}{\prod_{i=n-2}^{n} \phi_{i}^{-}} \int_{\varphi_{n-2}\left(\vartheta_{n-2}\right)}^{\varphi_{n-2}\left(\omega_{n-2}\right)} \int_{\varphi_{n-1}\left(\vartheta_{n-1}\right)}^{\varphi_{n-1}\left(\omega_{n-1}\right)} \int_{\varphi_{n}\left(\vartheta_{n}\right)}^{\varphi_{n}\left(\omega_{n}\right)} \psi_{\varphi_{n}\left(\alpha_{n}\right)}^{n}\left(\alpha_{n}\right) d \alpha_{n} d \alpha_{n-1} d \alpha_{n-2}
\end{gathered}
$$




$$
\leq \frac{1}{2^{3}}\left[\begin{array}{c}
\psi\left(\bigwedge_{k=1}^{n-3} \varphi_{k}\left(\alpha_{k}\right), \varphi_{n-2}\left(\vartheta_{n-2}\right), \varphi_{n-1}\left(\vartheta_{n-1}\right), \varphi_{n}\left(\vartheta_{n}\right)\right) \\
+\psi\left(\bigwedge_{k=1}^{n-3} \varphi_{k}\left(\alpha_{k}\right), \varphi_{n-2}\left(\omega_{n-2}\right), \varphi_{n-1}\left(\vartheta_{n-1}\right), \varphi_{n}\left(\vartheta_{n}\right)\right) \\
+\psi\left(\bigwedge_{k=1}^{n-3} \varphi_{k}\left(\alpha_{k}\right), \varphi_{n-2}\left(\vartheta_{n-2}\right), \varphi_{n-1}\left(\omega_{n-1}\right), \varphi_{n}\left(\omega_{n}\right)\right) \\
+\psi\left(\bigwedge_{k=1}^{n-3} \varphi_{k}\left(\alpha_{k}\right), \varphi_{n-2}\left(\omega_{n-2}\right), \varphi_{n-1}\left(\omega_{n-1}\right), \varphi_{n}\left(\vartheta_{n}\right)\right) \\
+\psi\left(\bigwedge_{k=1}^{n-3} \varphi_{k}\left(\alpha_{k}\right), \varphi_{n-2}\left(\vartheta_{n-2}\right), \varphi_{n-1}\left(\vartheta_{n-1}\right), \varphi_{n}\left(\omega_{n}\right)\right) \\
+\psi\left(\bigwedge_{k=1}^{n-3} \varphi_{k}\left(\alpha_{k}\right), \varphi_{n-2}\left(\omega_{n-2}\right), \varphi_{n-1}\left(\vartheta_{n-1}\right), \varphi_{n}\left(\omega_{n}\right)\right) \\
+\psi\left(\bigwedge_{k=1}^{n-3} \varphi_{k}\left(\alpha_{k}\right), \varphi_{n-2}\left(\vartheta_{n-2}\right), \varphi_{n-1}\left(\omega_{n-1}\right), \varphi_{n}\left(\omega_{n}\right)\right) \\
+\psi\left(\bigwedge_{k=1}^{n-3} \varphi_{k}\left(\alpha_{k}\right), \varphi_{n-2}\left(\omega_{n-2}\right), \varphi_{n-1}\left(\omega_{n-1}\right), \varphi_{n}\left(\omega_{n}\right)\right)
\end{array}\right] .
$$

So, using inductive method and taking into account $\psi_{\varphi_{n}\left(\alpha_{n}\right)}^{n}\left(\alpha_{n}\right):=\psi\left(\alpha_{1}, \ldots, \alpha_{n}\right)$, we get (7).

Remark 2.2. Taking into account Theorem 2.3, under the suitable conditions, then one can obtain HermiteHadamard type integral inequality for the functions in Definition 2.1.

Example 2.1. Let $\psi: \xi^{3} \rightarrow \mathbb{R}$ be a multidimensional general convex functions. Then

$$
\begin{gathered}
\psi\left(\frac{\varphi_{1}\left(\vartheta_{1}\right)+\varphi_{1}\left(\omega_{1}\right)}{2}, \frac{\varphi_{2}\left(\vartheta_{2}\right)+\varphi_{2}\left(\omega_{2}\right)}{2}, \frac{\varphi_{3}\left(\vartheta_{3}\right)+\varphi_{3}\left(\omega_{3}\right)}{2}\right) \\
\leq \frac{1}{\prod_{i=n-2}^{3}\left(\varphi_{i}\left(\omega_{i}\right)-\varphi_{i}\left(\vartheta_{i}\right)\right)} \int_{\varphi_{1}\left(\vartheta_{1}\right)}^{\varphi_{1}\left(\omega_{1}\right)} \int_{\varphi_{2}\left(\vartheta_{2}\right)}^{\varphi_{2}\left(\omega_{2}\right)} \int_{\varphi_{3}\left(\vartheta_{3}\right)}^{\varphi_{3}\left(\omega_{3}\right)} \psi\left(\left(\alpha_{1}, \alpha_{2}, \alpha_{3}\right)\right) d \alpha_{3} d \alpha_{2} d \alpha_{1} \\
\leq \frac{1}{2^{3}}\left[\begin{array}{c}
\psi\left(\varphi_{1}\left(\vartheta_{1}\right), \varphi_{2}\left(\vartheta_{2}\right), \varphi_{3}\left(\vartheta_{3}\right)\right)+\psi\left(\varphi_{1}\left(\omega_{1}\right), \varphi_{2}\left(\vartheta_{2}\right), \varphi_{3}\left(\vartheta_{3}\right)\right) \\
+\psi\left(\varphi_{1}\left(\vartheta_{1}\right), \varphi_{2}\left(\omega_{2}\right), \varphi_{3}\left(\vartheta_{3}\right)\right)+\psi\left(\varphi_{1}\left(\omega_{1}\right), \varphi_{2}\left(\omega_{2}\right), \varphi_{3}\left(\vartheta_{3}\right)\right) \\
\left.+\psi\left(\varphi_{1}\right), \varphi_{2}\left(\vartheta_{2}\right), \varphi_{3}\left(\omega_{3}\right)\right)+\psi\left(\varphi_{1}\left(\omega_{1}\right), \varphi_{2}\left(\vartheta_{2}\right), \varphi_{3}\left(\omega_{3}\right)\right) \\
+\psi\left(\varphi_{1}\left(\vartheta_{1}\right), \varphi_{2}\left(\omega_{2}\right), \varphi_{3}\left(\omega_{3}\right)\right)+\psi\left(\varphi_{1}\left(\omega_{1}\right), \varphi_{2}\left(\omega_{2}\right), \varphi_{3}\left(\omega_{3}\right)\right)
\end{array}\right] .
\end{gathered}
$$

Surely, according to Theorem 2.3 for $\mathrm{n}=3$, we get

$$
\begin{gathered}
\psi_{\varphi_{3}\left(\alpha_{3}\right)}^{3}\left(\varphi_{3}\left(\alpha_{3}\right)\right):=\psi\left(\varphi_{1}\left(\alpha_{1}\right), \varphi_{2}\left(\alpha_{2}\right), \varphi_{3}\left(\alpha_{3}\right)\right):=\psi\left(\left(\alpha_{1}, \alpha_{2}, \alpha_{3}\right)\right) ; \\
\tau_{i}(3):=\left\{\xi \in \mathbb{N}_{0}^{3}: \xi_{i} \leq 1,|\xi|=4-i\right\}, i=1,2,3,4 .
\end{gathered}
$$

Then

$$
\begin{aligned}
& \tau_{1}(3)=\{(1,1,1)\} ; \tau_{2}(3)=\{(0,1,1),(1,0,1),(1,1,0)\} \\
& \tau_{3}(3)=\{(0,0,1),(0,1,0),(1,0,0),\} ; \tau_{4}(3)=\{(0,0,0)\} .
\end{aligned}
$$

Consequently

$$
=\psi\left(\begin{array}{c}
\psi\left(\varphi_{1}\left(\vartheta_{1}\right), \varphi_{2}\left(\vartheta_{2}\right), \varphi_{3}\left(\vartheta_{3}\right)\right) \\
(1,1,1)\left(\varphi_{1}\left(\vartheta_{1}\right), \varphi_{2}\left(\vartheta_{2}\right), \varphi_{3}\left(\vartheta_{3}\right)\right) \\
+[(1,1,1)-(1,1,1)]\left(\varphi_{1}\left(\omega_{1}\right), \varphi_{2}\left(\omega_{2}\right), \varphi_{3}\left(\omega_{3}\right)\right)
\end{array}\right)
$$

for $\boldsymbol{\varphi}(\boldsymbol{\vartheta})=\left(\varphi_{1}\left(\vartheta_{1}\right), \varphi_{2}\left(\vartheta_{2}\right), \varphi_{3}\left(\vartheta_{3}\right)\right), \boldsymbol{\varphi}(\boldsymbol{v})=\left(\varphi_{1}\left(\omega_{1}\right), \varphi_{2}\left(\omega_{2}\right), \varphi_{3}\left(\omega_{3}\right)\right)$. Thus

$$
\sum_{\xi \in \tau_{1}(3)} \psi(\xi \boldsymbol{\varphi}(\boldsymbol{\vartheta})+(\mathbf{1}-\xi) \boldsymbol{\varphi}(\boldsymbol{\omega}))=\psi\left(\varphi_{1}\left(\vartheta_{1}\right), \varphi_{2}\left(\vartheta_{2}\right), \varphi_{3}\left(\vartheta_{3}\right)\right)
$$

Similarly by $\boldsymbol{\tau}_{2}(3), \boldsymbol{\tau}_{3}(3)$ and $\boldsymbol{\tau}_{4}(3)$, respectively, we get 


$$
\begin{aligned}
& \sum_{\xi \in \tau_{2}(3)} \psi(\xi \boldsymbol{\varphi}(\boldsymbol{\vartheta})+(\mathbf{1}-\xi) \boldsymbol{\varphi}(\boldsymbol{\omega}))=\psi\left(\varphi_{1}\left(\vartheta_{1}\right), \varphi_{2}\left(\omega_{2}\right), \varphi_{3}\left(\omega_{3}\right)\right) \\
& +\psi\left(\varphi_{1}\left(\vartheta_{1}\right), \varphi_{2}\left(\omega_{2}\right), \varphi_{3}\left(\vartheta_{3}\right)\right)+\psi\left(\varphi_{1}\left(\vartheta_{1}\right), \varphi_{2}\left(\vartheta_{2}\right), \varphi_{3}\left(\omega_{3}\right)\right) ; \\
& \sum_{\xi \in \tau_{3}(3)} \psi(\xi \boldsymbol{\varphi}(\boldsymbol{\vartheta})+(\mathbf{1}-\xi) \boldsymbol{\varphi}(\boldsymbol{\omega}))=\psi\left(\varphi_{1}\left(\omega_{1}\right), \varphi_{2}\left(\omega_{2}\right), \varphi_{3}\left(\vartheta_{3}\right)\right) \\
& +\psi\left(\varphi_{1}\left(\omega_{1}\right), \varphi_{2}\left(\vartheta_{2}\right), \varphi_{3}\left(\omega_{3}\right)\right)+\psi\left(\varphi_{1}\left(\vartheta_{1}\right), \varphi_{2}\left(\omega_{2}\right), \varphi_{3}\left(\omega_{3}\right)\right) ; \\
& \sum_{\xi \in \tau_{4}(3)} \psi(\xi \boldsymbol{\varphi}(\boldsymbol{\vartheta})+(\mathbf{1}-\xi) \boldsymbol{\varphi}(\boldsymbol{\omega}))=\psi\left(\varphi_{1}\left(\omega_{1}\right), \varphi_{2}\left(\omega_{2}\right), \varphi_{3}\left(\omega_{3}\right)\right) .
\end{aligned}
$$

Finally, using all of the above equalities in (7), we obtain the desired result in this example.

\section{Conclusions and Recommendations}

The main contribution of this study to the literature is the introduction of general convexity for multidimensional functions. As a result, some Hermite-Hadamard type integral inequalities for these functions were obtained mathematically. We hope that with the methods used in this study, original results can be obtained for different functions.

\section{Acknowledgements}

The author would like to thank to Assoc. Prof. Erhan SET from Ordu University and Assoc. Prof. İmdat İşCAN from Giresun University owing to enlightening ideas.

\section{Kaynaklar}

Cristescu, G., 2004. Hadamard type inequalities for $\varphi$ convex functions: Annals of the University of Oradea, Fascicle of Management and Technological Engineering, CD-Rom Edition, III(XIII).

De la Cal, J. and Carcamo, J., 2006. Multidimensional Hermite-Hadamard inequalities and the convex order. Journal of Mathematical Analysis and Applications, 324, 248-261.

Dragomir, S.S., 2015. Inequalities of Jensen Type for $\varphi$-Convex Functions. Fasciculi Mathematici, 5, $35-52$.

Dragomir, S.S, 2001. On Hadamards inequality for convex functions on the coordinates in a rectangle from the plane. Taiwanese Journal of Mathematics, 4, 775-788.

Ellahi, H., Farid, G., and Rehman, A.U., 2015. Hadamard's Inequality for s-convex function on n-coordinates, Proceedings of 1st ICAM Attock, Pakistan.
Hadamard, J., 1893. Étude sur les propriétés des fonctions entières et en particulier d.une function considerée par Riemann. Journal de Mathématiques Pures et Appliquées, 58, 171215.

Latif M.A. and Alomari, M., 2009. On Hadamard-type inequalities for $\mathrm{h}$-convex functions on the coordinates. International Journal of Mathematical Analysis, 33, 1645-1656.

Martinez-Legaz, J.E. and Singer, I., 1998. On $\varphi$ convexity of convex functions. Linear Algebra and its Applications, 278, 163-181.

Okur, N., İşcan, İ., and Yüksek Dizdar, E., 2019. Hermite-Hadamard Type Inequalities for pConvex Stochastic Processes. An International Journal of Optimization and Control: Theories and Applications, 9(2), 148-153.

Okur, N., 2019. Multidimensional General Convexity for Stochastic Processes and Associated with Hermite-Hadamard Type Integral Inequalities. Thermal Science, doi: 10.2298/TSCI190622361O.

Okur, N. and Yalçın, F.B., 2019. Two-Dimensional Operator Harmonically Convex Functions and Related Generalized Inequalities. Turkish Journal of Science, 5(1), 30-38.

Sarikaya, M.Z., Büyükeken, M., and Kiriş, M.E., 2015. On Some Generalized Integral Inequalities for $\varphi$-Convex Functions. Studia Universitatis Babeş-Bolyai Mathematica, 60(3), 367-377.

Set, E., Sarıkaya, M. Z., and Akdemir, A. O., 2014. Hadamard type inequalities for $\varphi$-convex functions on the co-ordinates. Tbilisi Mathematical Journal, 7(2), 51-60.

Shaikh A.A., Iqbal, A., and Mondal, C.K., 2018. Some Results on $\varphi$-Convex Functions and Geodesic $\Phi$-Convex Functions. Differential GeometryDynamical Systems, 20, 159-169. 
Syau, Y.R. and Lee, E.S., 2005. Some Properties of EConvex Functions. Applied Mathematics Letters, 18, 1074-1080.

Viloria, J.M. and Cortez, M.V., 2018. HermiteHadamard type inequalities for harmonically convex functions on $\mathrm{n}$-coordinates. Applied Mathematics and Information Sciences Letter 6(2), 1-6.
Yalçın, F.B., 2019. On Some Generalized Inequalities for Two-Dimensional $\varphi$-Convex Functions, Journal of Contemporary Applied Mathematics, 9(1), 88-102.

Youness, E.A., 1999. E-Convex Sets, E-Convex Functions and E-Convex Programming. Journal of Optimization Theory and Applications, 102, 439-450. 